\title{
\begin{tabular}{|c|l|}
\hline IV Simpósio Paranaense de Modelagem, & Artigo: \\
$\begin{array}{c}\text { Simulaçãoe Controle de Processos } \\
\text { ISSN : 1984-7521 }\end{array}$ & Páginas: 113 - 119
\end{tabular}
}

\section{MODELAGEM FRACIONÁRIA APLICADA A REATORES TUBULARES NÃO IDEAIS}

\author{
Wesley P. do Carmo*, Alexandre F. Santos, Marcelo Kaminski Lenzi \\ Universidade Federal do Paraná - UFPR - PR, *wesley.pardinho@gmail.com
}

\begin{abstract}
Resumo - O objetivo deste trabalho foi propor um modelo fracionário capaz de descrever o comportamento dos reatores tubulares não ideais, em especial o comportamento dos reatores tubulares de fluxo laminar (LFR). Para isso, generalizou-se a equação de projeto dos reatores tubulares de fluxo empistonado (PFR), obtendo-se o modelo fracionário para reatores tubulares. Em seguida, demostrou-se que a equação do PFR é um caso especial do modelo fracionário $(\boldsymbol{\alpha}=\mathbf{1})$ e que o modelo proposto gera perfis de conversão condizentes com aqueles esperados para reatores não ideais. Por fim, aplicou-se o modelo fracionário na descrição dos dados simulados de reatores de fluxo laminar, obtendo-se uma ordem de derivada ótima $\boldsymbol{\alpha}=\mathbf{0 , 8 2 0 2} \pm \mathbf{0 , 0 0 7 9}$ e coeficiente de correlação $\boldsymbol{R}^{2}>0,99$, evidenciando que o modelo fracionário é adequado para a descrição do comportamento de reatores tubulares não ideais. Além disso, os dados numéricos mostram que o modelo é estatisticamente consistente e que os reatores tubulares de fluxo laminar são descritos por um modelo de ordem fracionária.

Keywords: Reatores tubulares não ideais, cálculo fracionário, reatores tubulares de fluxo laminar (LFR).
\end{abstract}

\section{Introdução}

Os reatores tubulares ideais (PFR) são amplamente conhecidos, estudados e possuem literatura própria[1], [2] . No entanto, são idealizações imaginadas para facilitar sua modelagem matemática sendo que, na prática, devido a flutuações no processo e/ou erro de projeto nem todos os reatores se comportam de acordo com o modelo ideal.

Por outro lado, os reatores tubulares reais não possuem vasta literatura e, em geral, sua modelagem depende da obtenção da construção de perfis de tempo de residência experimentais[1].

Desta forma, o objetivo desse trabalho é propor um modelo, com base no cálculo fracionário, capaz de descrever o comportamento de reatores tubulares não ideais e, de forma mais específica, busca-se descrever o comportamento dos reatores tubulares de fluxo laminar (LFR).

\section{$O$ reator de fluxo empistonado $(P F R)$}

O reator tubular de fluxo empistonado (PFR) é uma idealização dos reatores reais. Sua modelagem é obtida a partir de um balanço mássico diferencial e possui como hipóteses principais: 1- escoamento totalmente turbulento; 2 - não há difusão no sentido axial do escoamento[3]. É amplamente utilizado, pois é o modelo de reator que apresenta a maior conversão para um dado volume[4]. É descrito matematicamente pela Eq. (1).

$$
\frac{d V}{d X_{A}}=\frac{F_{A 0}}{\left(-r_{A}\right)}
$$

Onde: $V$ é o volume do reator; $X_{A}$ é a conversão do reagente $\mathrm{A} ; F_{A 0}=Q . C_{A 0}$ é o fluxo molar dado pelo produto entre a vazão volumétrica e a concentração molar inicial da espécie $\mathrm{A} ;\left(-r_{A}\right)$ é a equação cinética.

\section{4 e 15 de março de 2019 Curitiba - Paraná}


A principal limitação do reator PFR é a manutenção do fluxo empistonado, ou seja, a manutenção do regime turbulento ao longo de todo o reator[1]. Sendo assim, como dito anteriormente, devido a flutuações no processo e/ou por falha de design, pode se operar o reator em regime laminar e, neste caso, a conversão será menor do que aquela prevista através da Eq. (1). Neste caso, pode-se obter a conversão média de um reator de fluxo laminar através do modelo de fluxo segregado. Para uma cinética de primeira ordem, a conversão média é dada pela Eq. (2)[1].

$$
X_{A}=1-\int_{\tau / 2}^{\infty} \frac{e^{-K t}}{t^{3}} \tau^{2} d t
$$

Onde: $\tau$ é o tempo espacial, dado por $\tau=V / Q ; t$ é o tempo de reação; $K$ é a constante cinética.

\section{Metodologia}

\section{A definição de Caputo da derivada fracionária}

Após a realização do primeiro congresso, a respeito do cálculo fracionário, na Universidade de New Haven em 1974, surgiram muitas aplicações em pesquisas cientificas [5], e muitos autores demonstraram sua enorme capacidade como ferramenta para descrever e solucionar problemas práticos.[6]-[13].

Como consequência, foram desenvolvidas distintas abordagens para a solução dos mais variados problemas e estão disponíveis diferentes definições de derivada fracionária. A definição de derivada fracionária, proposta por M. Caputo [14] é dada pela Eq. (3).

$$
\begin{aligned}
{ }_{a}^{c} D_{t}^{\alpha} f(t)= & \frac{1}{\Gamma(n-\alpha)} \int_{a}^{t} \frac{f^{n}(\varepsilon) d \varepsilon}{(t-\varepsilon)^{\alpha+1-n}} \\
& n-1 \leq \alpha<n
\end{aligned}
$$

Devido à complexidade da definição de derivada fracionária, frequentemente faz-se necessário a solução numérica das equações obtidas. Desta forma, a Eq. (4) apresenta a aproximação numérica da derivada fracionária de Caputo[15].

$$
{ }_{a}^{C} D_{t}^{\alpha} f(t)=\frac{1}{h^{\alpha}} \sum_{k=0}^{N} w_{k}^{(\alpha)} f(t-k \cdot h)-\sum_{k=0}^{n-1} \frac{f^{(k)}(a)}{\Gamma(k-\alpha+1)}(t-a)^{k-\alpha}
$$

Onde: $w_{k}^{(\alpha)}$ são pesos calculados através da relação recursiva dada pela Eq.(5); $h$ é o passo; $N$ é o número de pontos tal que $N=t / h$.

$$
\begin{gathered}
w_{0}^{(\alpha)}=1 \\
w_{k}^{(\alpha)}=\left(1-\frac{\alpha+1}{k}\right) w_{k-1}^{(\alpha)}
\end{gathered}
$$

Ajuste dos parâmetros e incertezas paramétricas 
O método de ajuste e determinação dos parâmetros dos modelos se baseia na técnica de mínimos quadrados. Para isso, formula-se o problema de otimização conforme demonstra-se a seguir.

Primeiramente, os parâmetros do modelo foram ajustados através da minimização da Eq. (6) utilizando o método simplex [16]

$$
F o b=\sum_{i=1}^{N_{y}}\left[y_{i}^{\text {exp }}-y_{i}^{\text {modelo }}(\theta)\right]^{2}
$$

Onde, $y_{i}^{\text {exp }}$ é o valor de referência, $y_{i}^{\text {modelo }}(\theta)$ é o valor da função calculada através da equação do modelo e $\theta$ é o vetor de parâmetros ajustáveis.

As incertezas paramétricas foram determinadas para demonstrar a relevância estatística de cada parâmetro do modelo com $95 \%$ de confiança e, para isso, faz-se necessário alguns cálculos matriciais. De início, estima-se a matriz de variância experimental, admitindo-se que o experimento fora bem feito, através da Eq. (7).

$$
V_{\text {exp }}=\sigma_{\text {exp }}^{2} \cdot I_{N_{y}}
$$

Onde: $\sigma_{\text {exp }}^{2}$ é a variância experimental estimada, $N_{y}$ é o número de pontos, $N_{\theta}$ é o número de parâmetros estimados e $I_{N_{y}}$ é a matriz identidade de ordem $N_{y}$.

$$
\sigma_{\text {exp }}^{2}=\frac{\sum_{i=1}^{N_{y}}\left[y_{i}^{\text {exp }}-y_{i}^{\text {modelo }}(\theta)\right]^{2}}{N_{y}-N_{\theta}}
$$

A matriz de covariância paramétrica $\left(V_{\theta}\right)$ é então calculada através da Eq. (9).

$$
V_{\theta}=H_{\theta}^{-1} G_{y} V_{\exp } G_{y}^{T}\left(H_{\theta}^{-1}\right)^{T}
$$

A matriz $G_{y}$ é dada pela Eq, (10) e a matriz hessiana $\left(H_{\theta}\right)$ é dada pela Eq. (11) e foram calculadas numericamente por diferenças finitas centrais.

$$
\begin{gathered}
G_{y}=\left[\begin{array}{cccc}
\frac{\partial^{2} F o b}{\partial \theta_{1} \partial y_{1}^{\text {exp }}} & \frac{\partial^{2} F o b}{\partial \theta_{1} \partial y_{2}^{\text {exp }}} & \cdots & \frac{\partial^{2} F o b}{\partial \theta_{1} \partial y_{N_{\gamma}}^{\text {exp }}} \\
\frac{\partial^{2} F o b}{\partial \theta_{2} \partial y_{1}^{e x p}} & \frac{\partial^{2} F o b}{\partial \theta_{2} \partial y_{2}^{e x p}} & \cdots & \frac{\partial^{2} F o b}{\partial \theta_{2} \partial y_{N_{y}}^{\text {exp }}} \\
\vdots & \vdots & \ddots & \vdots \\
\frac{\partial^{2} F o b}{\partial \theta_{N_{\theta}} \partial y_{1}^{e x p}} & \frac{\partial^{2} F o b}{\partial \theta_{N_{\theta}} \partial y_{2}^{e x p}} & \cdots & \frac{\partial^{2} F o b}{\partial \theta_{N_{\theta}} \partial y_{N_{y}}^{\text {exp }}}
\end{array}\right] \\
H_{\theta}=\left[\begin{array}{cccc}
\frac{\partial^{2} F o b}{\partial \theta_{1}^{2}} & \frac{\partial^{2} F o b}{\partial \theta_{1} \partial \theta_{2}} & \cdots & \frac{\partial^{2} F o b}{\partial \theta_{1} \partial \theta_{N_{\theta}}} \\
\frac{\partial^{2} F o b}{\partial \theta_{1} \partial \theta_{2}} & \frac{\partial^{2} F o b}{\partial \theta_{2}^{2}} & \cdots & \frac{\partial^{2} F o b}{\partial \theta_{2} \partial \theta_{N_{\theta}}} \\
\vdots & \vdots & \ddots & \vdots \\
\frac{\partial^{2} F o b}{\partial \theta_{1} \partial \theta_{N_{\theta}}} & \frac{\partial^{2} F o b}{\partial \theta_{2} \partial \theta_{N_{\theta}}} & \cdots & \frac{\partial^{2} F_{o b}}{\partial \theta_{N_{\theta}}^{2}}
\end{array}\right]
\end{gathered}
$$


Por fim, a incerteza paramétrica $(\Delta \theta)$ é dada pela Eq. (12).

$$
\Delta \theta_{i}=V_{\theta}(i, i) t(p, v)
$$

Onde $t(p, v)$ é o valor da distribuição t-student $\operatorname{com} p=0.95$ e $v=N_{\gamma}-N_{\theta}$.

\section{Resultados e discussões}

Postula-se que o modelo fracionário é capaz de descrever o comportamento dos reatores tubulares não ideais. Para tanto, generaliza-se a equação do PFR, Eq. (1), utilizando a derivada fracionária de Caputo. A Eq. (13) apresenta o modelo fracionário de reatores não ideais.

$$
{ }_{0}^{C} D_{t}^{\alpha} V\left(X_{A}\right)=\frac{F_{A 0}}{\left(-r_{A}\right)}
$$

Reescrevendo-se a Eq. (13) e rearranjando, na forma da Eq. (14), pode-se obter a solução analítica do modelo.

$$
\begin{gathered}
\text { a. }{ }_{0}^{C} D_{t}^{\alpha} V\left(X_{A}\right)=f\left(X_{A}\right) \\
a=\frac{1}{F_{A 0}} ; f\left(X_{A}\right)=\frac{1}{\left(-r_{A}\right)}
\end{gathered}
$$

Note que, nesta forma, a solução - Eq. (15) - é dada por uma equação integrodiferencial e depende da cinética de reação. Este é um fator que limita a obtenção de expressões analíticas e, por vezes, é preferível partir para obtenção da solução numérica do problema.

$$
V\left(X_{A}\right)=\frac{F_{A 0}}{\Gamma(\alpha)} \int_{0}^{X_{A}} \frac{f(\varepsilon) d \varepsilon}{\left(X_{A}-\varepsilon\right)^{1-\alpha}}
$$

Onde: $\Gamma(\alpha)$ é a função gama e $\varepsilon$ é uma variável auxiliar de integração.

Analisando a solução obtida, é fácil perceber que a equação do PFR, Eq. (1), é um caso especial da Eq. (15). Para obtê-la, basta tomar o limite quando $\alpha \rightarrow 1$ e fazer a substituição de variável: $\varepsilon=X_{A} \therefore d \varepsilon=d X_{A}$.

$$
\lim _{\alpha \rightarrow 1} V\left(X_{A}\right)=\frac{F_{A 0}}{\Gamma(1)} \int_{0}^{X_{A}} \frac{f(\varepsilon) d \varepsilon}{\left(X_{A}-\varepsilon\right)^{1-1}}=F_{A 0} \int_{0}^{X_{A}} \frac{d X_{A}}{\left(-r_{A}\right)}=V_{P F R}\left(X_{A}\right)
$$

Supondo-se uma lei cinética de primeira ordem, dada pela Eq. (17). Pode-se obter os perfis de conversão dos reatores para diversas ordem de derivada fracionária.

$$
\left(-r_{A}\right)=K \cdot C_{A}=K \cdot C_{A 0}\left(1-X_{A}\right)
$$

No entanto, devido à complexidade do problema e a impossibilidade de se obter uma solução analítica da integral da Eq. (15), todos os resultados simulados foram obtidos através da solução numérica da Eq. (13) de acordo com a aproximação numérica da derivada de Caputo, dada pela Eq. (4). Sendo assim, substituindo-se a Eq. (4) na Eq. (13) e rearranjando, obtém-se o algoritmo de solução numérica, Eq. (18). 


$$
V\left(X_{A_{N}}\right)=\frac{h^{\alpha} \cdot Q}{K\left(1-X_{A_{N}}\right)}-\sum_{k=1}^{N} w_{k}^{(\alpha)} V\left(X_{A_{N-k}}\right)
$$

Os perfis de conversão simulados para diferentes ordens de derivada, admitindo-se: $Q=1 \mathrm{~m}^{3} / \mathrm{s}$ e $K=1 \mathrm{~s}^{-1}$; são apresentados na Figura 01 .

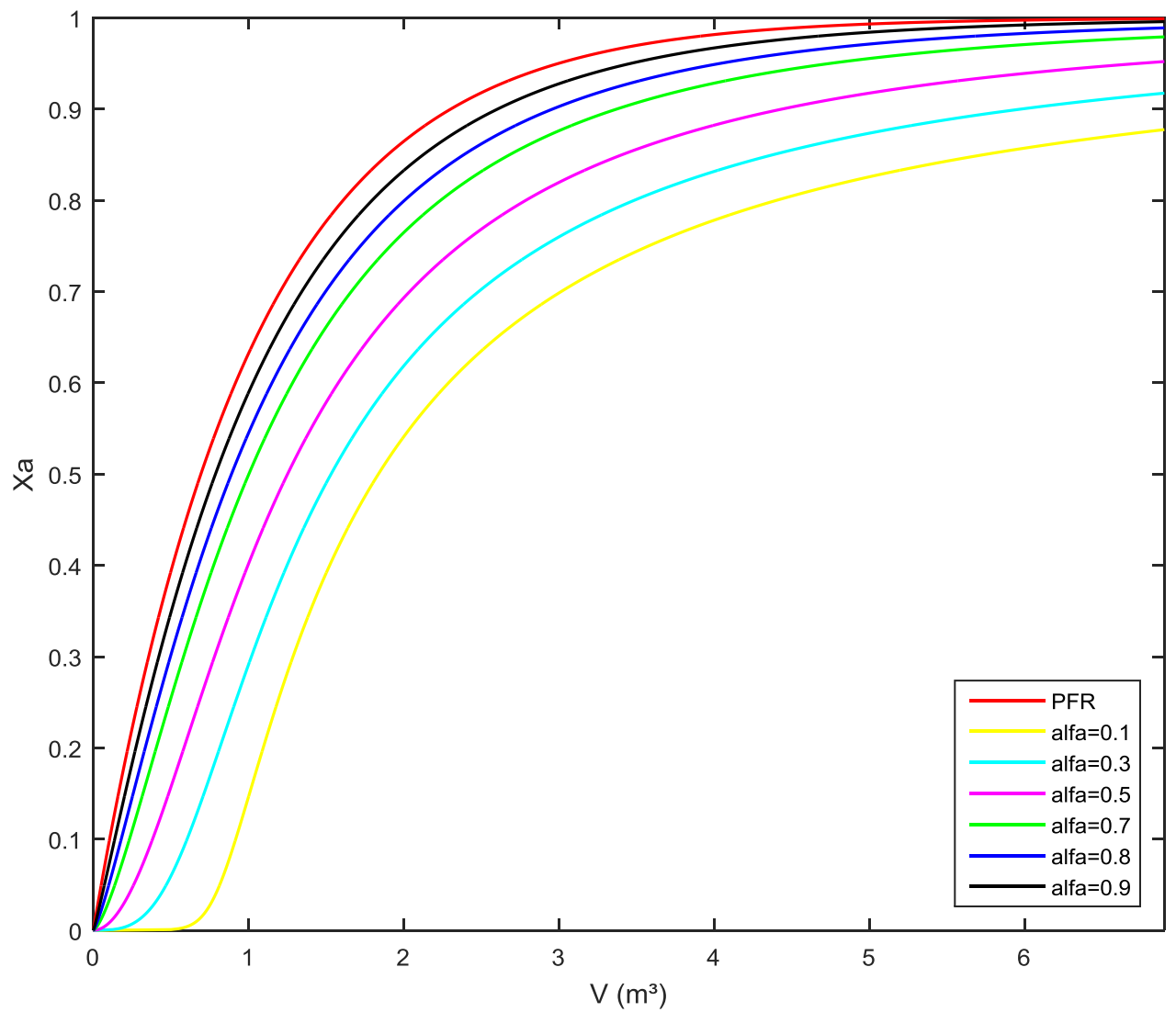

Figura 1 - Perfis de conversão para diferentes ordens de derivada.

Analisando-se os resultados apresentados na Figura 01, nota-se que os perfis de conversão crescem com o aumento da derivada e, assim como previsto na Eq. (16), tendem ao perfil de conversão do PFR quando a ordem da derivada tende a 1 . Tendo em vista que o reator PFR é um caso idealizado e, portanto, o limite de conversão teórica de uma reação para um mesmo volume. É natural que a conversão para reatores não ideais seja menor do que aquela obtida no reator PFR[1]. Desta forma, os resultados apresentados dão suporte para a hipótese de que o modelo fracionário é adequado para a descrição do comportamento de reatores não ideais.

Para testar essa hipótese, buscou-se descrever o comportamento de reatores tubulares de fluxo laminar (LFR) calculados a partir do modelo de segregação, Eq. (2). De forma semelhante ao feito anteriormente, admitiu-se uma cinética de primeira ordem, dada pela Eq. (17), e a integral presente na Eq. (2) foi resolvida através do método de quadratura de Gauss-Kronrod[17].

Concomitantemente, fez-se a otimização (conforme metodologia de ajuste de parâmetros apresentada anteriormente) da ordem do modelo fracionário, Eq. (13), para que esse representasse bem os dados simulados do reator LFR. Os resultados obtidos são apresentados na Figura 2. 


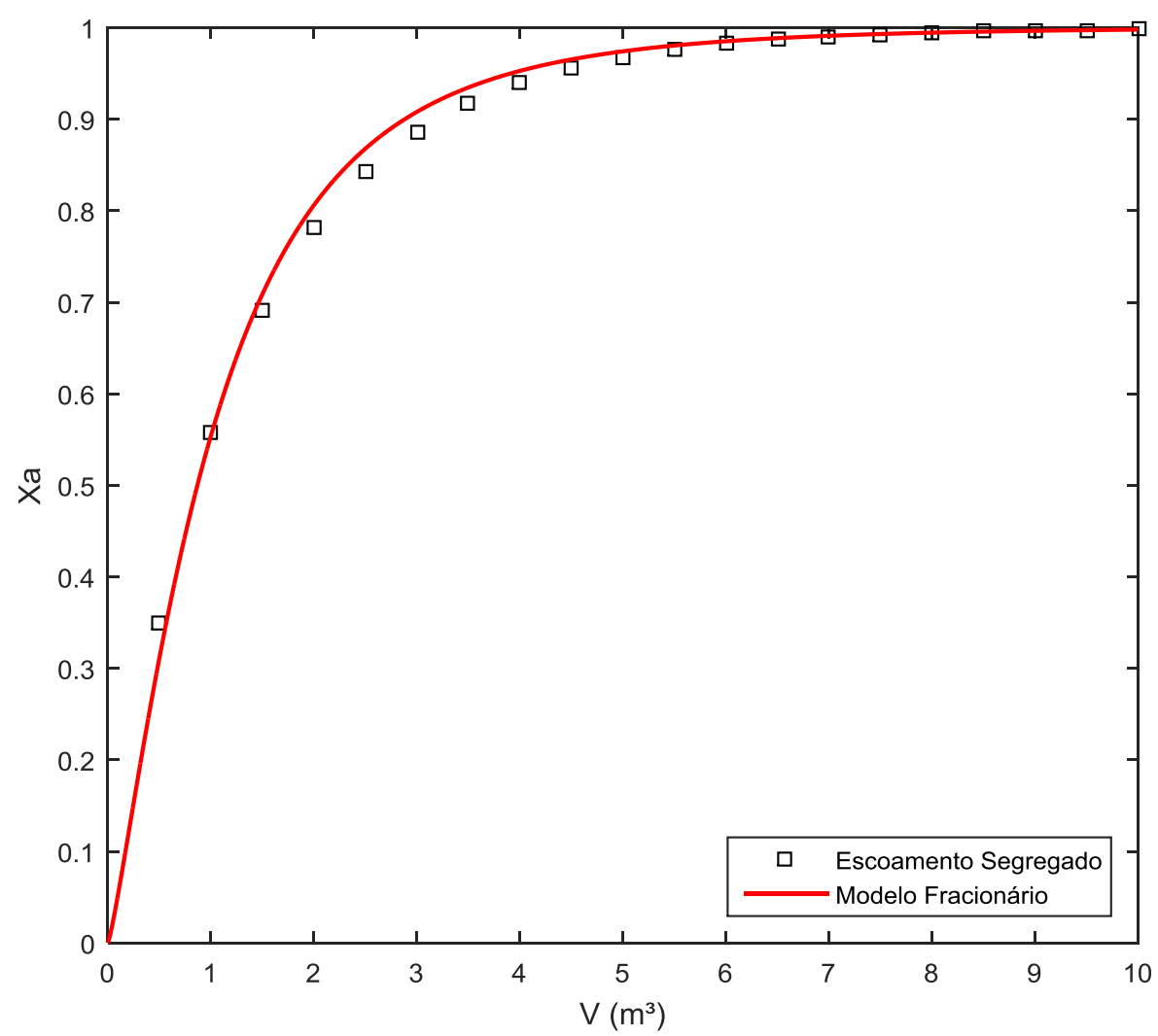

Figura 2 - Dados de conversão para o reator LFR e ajuste do modelo fracionário

Do processo de otimização resulta.

$$
\begin{gathered}
\alpha=0,8202 \pm 0,0079 \\
R^{2}=0,9916
\end{gathered}
$$

Da analise da Figura 2 e dos resultados numéricos, percebe-se que há um bom ajuste do modelo fracionário aos dados obtidos para o reator LFR, prova disso é o coeficiente de correlação $R^{2}>0,99$. Além disso, obteve-se o valor da ordem da derivada que melhor ajusta o modelo e sua incerteza paramétrica, provando se tratar de um fenômeno fracionário $(\alpha \neq 1)$ e estatisticamente consistente.

\section{Conclusões}

Fora proposto a utilização do cálculo fracionário para a descrição do comportamento de reatores tubulares reais (não ideais). Para isso, primeiramente generalizou-se a equação de projeto do reator PFR utilizando a definição de Caputo da derivada fracionário.

De posse do modelo, foi possível demostrar que a equação do PFR é um caso especial do modelo fracionário, caso esse quando $\alpha \rightarrow 1$. Posteriormente, foram gerados perfis de conversão para diferentes ordens de derivada, evidenciando que o modelo fracionário prevê conversões inferiores aquelas obtidas no reator PFR. Este resultado é bastante condizendo com o comportamento esperado de reatores não ideais.

Por fim, aplicou-se o modelo fracionário na descrição do comportamento de reatores de fluxo laminar (LFR). Os resultados apresentados mostram que o modelo é eficiente na descrição desses reatores, com $\alpha=0,8202 \pm 0,0079$ e coeficiente de correlação $R^{2}>0,99$. Os resultados comprovam que o modelo é estatisticamente consistente e que o fenômeno é, de fato, fracionário. 


\section{Referências}

[1] H. S. Fogler, Elements of chemical reaction engineering, 3rd Editio. New Jersey: Prentice Hall, Inc, 1999.

[2] O. Levenspiel, Chemical Reaction Engineering, 3rd Editio. New York, NY, USA: John Wiley \& Sons, 1999.

[3] A. Bermúdez, N. Esteban, J. L. Ferrín, J. F. Rodríguez-calo, and M. R. Sillerodenamiel, "Identification problem in plug-flow chemical reactors using the adjoint method," Comput. Chem. Eng., vol. 98, pp. 80-88, 2017.

[4] S. Ramji, A. Vir, and S. Pushpavanam, "Two phase gas-liquid strati fi ed laminar fl ows in tubular reactors sustaining liquid phase reactions," Chem. Eng. J., vol. 356, no. September 2018, pp. 609-621, 2019.

[5] E. C. Oliveira and J. A. T. Machado, "A review of definitions for fractional derivatives and integral," Math. Probl. Eng., vol. 2014, no. 1940, 2014.

[6] A. D. Obembe, H. Y. Al-Yousef, M. E. Hossain, and S. A. Abu-Khamsin, "Fractional derivatives and their applications in reservoir engineering problems: A review," J. Pet. Sci. Eng., vol. 157, no. July, pp. 312-327, 2017.

[7] H. Xu and X. Jiang, "Creep constitutive models for viscoelastic materials based on fractional derivatives," Comput. Math. with Appl., vol. 73, no. 6, pp. 13771384, 2017.

[8] Y. Zhang, H. G. Sun, H. H. Stowell, M. Zayernouri, and S. E. Hansen, "A review of applications of fractional calculus in Earth system dynamics," Chaos, Solitons and Fractals, vol. 102, no. December 2016, pp. 29-46, 2017.

[9] A. Jaques, C. Da Silva, N. Duru, and T. Seal, "Fractional calculus application for diffusion controlled leaching column testing," Int. J. Miner. Process., vol. 169, pp. 185-187, 2017.

[10] L. Vinnett, M. Alvarez-Silva, A. Jaques, F. Hinojosa, and J. Yianatos, "Batch flotation kinetics: Fractional calculus approach," Miner. Eng., vol. 77, pp. 167$171,2015$.

[11] J.-L. Battaglia, O. Cois, L. Puigsegur, and A. Oustaloup, "Solving an inverse heat conduction problem using a non-integer identified model," Int. J. Heat Mass Transf., vol. 44, no. 14, pp. 2671-2680, 2001.

[12] Q. Yang, D. Chen, T. Zhao, and Y. Chen, "Fractional calculus in image processing: A review," Fract. Calc. Appl. Anal., vol. 19, no. 5, pp. 1222-1249, 2016.

[13] C. Ionescu, A. Lopes, D. Copot, J. A. T. Machado, and J. H. T. Bates, "The role of fractional calculus in modeling biological phenomena: A review," Commun. Nonlinear Sci. Numer. Simul., vol. 51, pp. 141-159, 2017.

[14] M. Caputo, "Linear models of dissipaton whose Q is almost frequency independet II," Geophys. J. R. astr. Soc., vol. 13, pp. 529-539, 1967.

[15] I. Podlubny, Fractional Differential Equations, vol. 198. Elsevier, 1999.

[16] G. B. Dantzig, "A History of Scientific Computing," S. G. Nash, Ed. New York, NY, USA: ACM, 1990, pp. 141-151.

[17] A. S. Kronrod, Nodes and weights of quadrature formulas : sixteen-place tables. New York: Consultants Bureau, 1965. 